\title{
Host Pathogen Interaction-Mycobacterium and Host Macrophages
}

\author{
Shalini Sharma* and Gursimran Filia \\ Animal disease research centre, GADVASU, Ludhiana, 141012, Punjab, India \\ *Corresponding author
}

Keywords

MTBC, TLR,

MHC,

Macrophages,

Granuloma

Article Info

Accepted:

18 January 2020

Available Online:

10 February 2020
A B S T R A C T

Mycobacterium tuberculosis (MTB) is an intracellular parasite that mainly attacks macrophages and establish a niche to survive in macrophages. It can become a long-term infection in humans and animals, causing a series of pathological changes and clinical manifestations. In this article, we summarizes how mycobacterium evades innate immunity and establish a niche in the host cell macrophages. Firstly it evades from the immune detection by masking, it manipulates the TLR response, manipulation of the antigen presentation by MHC, inhibition of phagosomal maturation in which Iron, Hydrogen and Calcium ions down regulation play an important role and phagosomally sosomal fusion, Inhibition of acidification of Phagolysosomes, Inhibition of Oxidative Stress and the Function of Reactive Oxygen and Reactive Nitrogen Intermediates, and inhibition of the apoptosis and autophagy of macrophages. A thorough understanding of this host pathogen interaction and thus immune escape of Mycobacterium an intracellular pathogen is of major importance for the prevention and diagnosis of bovine tuberculosis.

\section{Introduction}

Mycobacterium tuberculosis and Mycobacterium bovis are the most commonly encountered members of the Mycobacterium tuberculosis complex (MTBC) species worldwide. The former is the main causative agent of $\mathrm{TB}$ in humans, and the latter is responsible for the disease mainly in animals $(1,2)$. Transmission of tuberculosis caused by both pathogens can occur from human to animals and vice versa $(3,4)$.In developing countries like India the dietary habit of people, close physical contact between humans and animals, rise in the incidence of immunosuppressive diseases, and inadequate disease control measures in animals and humans facilitate the transmission of the disease between animals and humans (5). Since both are the intracellular pathogens so 
follow same strategies of immune escape. Diagnostic methods such as tuberculin skin test (TST) and cell-mediated immune response-dependent approaches were developed based on the current understanding of the mechanisms that contribute to the establishment of persistent infection (6). The latest developments in understanding the cellular, biochemical, and molecular mechanisms that are employed for the establishment of latent stage by Mtb are discussed in this review.

MTB is the pathogen that causes tuberculosis. Healthy ones can be infected via the respiratory tract, the digestive tract, damaged skin and mucous membranes (7). Inhalation of droplets containing MTB is the main route of infection. So how this pathogen interacts with the host cell macrophages and downregulates the host immune response is discussed in this review.

\section{Immune escape mechanism of Mycobacterium}

\section{Evading immune detection by masking}

Mycobacteria adopt multiple strategies to avoid the attack from macrophages its cell membranes contain methyl branched-chain fatty acids that protect them from host enzymes and enable them to escape immune responses (8). They express surface lipids such as phthioceroldimycoceroserate, which can mask the pathogen-associated molecular patterns (PAMPs), thereby going "unnoticed" by the innate immune system (9). In the upper airway where a constant and heavy recruitment of macrophages occurs due to the presence of TLR stimulating bacteria, Mtb adopts a different immune evasion strategy by forming small infection droplets that allow them to be delivered directly into the alveolar spaces of the lower lung, which anchorages a few microbicidal macrophages (10).
Interestingly, Mycobacterium is capable of modulating PPAR $\gamma$ to assist its pathogenesis by manipulating its function in lipid metabolism: mycobacteria use the hosts' lipids for intracellular survival and replication (11). MTB infection increases the expression of peroxisome proliferator-activated receptor gamma PPAR $\gamma$ via mechanisms including pattern recognition receptor activation, overexpression and activation resulting in increasing lipid droplet formation and downregulation of the macrophage response.

\section{Manipulating the TLR responses}

In the macrophages, which are the crucial niche for replication, Mtb interacts with various receptors to initiate phagocytosis. Despite the bactericidal properties of the macrophages, Mtb employs phagocytosis as a primary mode of gaining entry to establish the niche. Recognition of Mtb through its cell wall glycolipids involves the formation of TLR heterodimers (12). These heterodimers are formed for better ligand and receptor interactions. Exposure of THP-1 cells to Mtb cell wall components results in the de novo synthesis of TLR4, thereby decreasing the production of Th1 cytokines (13). Although Myd88-dependent signaling of TLRs is well established in mycobacterial pathogenesis, recent studies indicate independent roles for Mal (the TLR adaptor) and Myd88. TLR-4 by TRIF/TRAM pathway which is independent of Myd88 signaling increases the production of Th2 cytokines such as TGF- $\beta$, IL-4, IL-10 which attenuate the defensive cytokine response.

Interaction of Mtb cell wall components with TLRs modulates a number of events that include antigen presentation (14), phagolysosomalfusion (15), apoptosis of macrophages (12), and production of reactive oxygen and nitrogen intermediates (16). 
Manipulating the antigen presentation by MHC

The TLR2-dependent surface expression of MHC class II receptor and their antigenpresenting ability was found to be inhibited by either Mtb infected or 19-kDa lipoprotein (LpqH) exposed macrophages $(12,17)$. Class II transactivator (CIITA), a TLR-2-dependent regulator of MHC class II a, b, invariant chains contributes to antigen processing and its expression was found to be decreased during Mtb infection $(18,19)$.Mtb also inhibits the expression of genes involved in MHC class II processing and presentation $(20,21)$ and the posttranslational function of these molecules.

\section{Phagosomal maturation Phagosomal/lysosomal fusion arrest}

and

Repeated stimulation of TLRs by the Mtb components such as mannosylated-LAM (ManLAM) and PIM causes phagosomal maturation arrest allowing persistence of mycobacteria inside the phagosome (22).Among the successful strategies adopted by Mtb to establish a niche in the host, inhibition of macrophage maturation is best characterized. The mycobacterial products (ManLAM, trehalosedimycolate, and sulfolipids), phosphatase SapM, kinase PknG, and early secretory antigenic target-6 (ESAT6) have been implicated in the $\mathrm{Ca}+$ surge that modulates the calmodulin- and $\mathrm{Ca} 2+/$ calmodulin- dependent kinase IIdependent delivery of early endosomalautoantigen $1 \quad(23,24), \quad$ EEA1 localizes exclusively to early endosomes and has an important role in endosomal trafficking. EEA1 binds directly to the phospholipid phosphatidylinositol 3phosphate through its C-terminal FYVE domain.EEA1 is necessary for the delivery of lysosomal hydrolases and vacuolar $\mathrm{H}+-$ ATPases into phagosomes. ManLAM blocks
ESAT-6 recruitment by inhibiting PI3K hVPS34 to block PIP3 production. SapM also decreases PIP3 production. Phosphatidylinositol 3-phosphate (PI3P) is an important component of the macrophage cell membrane located on the early endosome and phagosome surface. PI3P helps in the fusion between phagosomes and lysosomes. Down regulation of PI3P by mycobacterium suppresses the process of fusion. LAM reduce the levels of Rab5 the gene responsible for EEA1 recruitment and the gene responsible for phagososme and lysosome fusion.Mtb also disrupts the scaffolding of endosomes required for phagosome-endosome interactions leading to delay in phagosomal maturation $(25,26)$.

PKnG is a protein similar to protein kinase in eukaryotes. PKnG enhances MTB metabolism, growth rate, virulence and drug resistance. PKnG secreted by MTB prevents the fusion of phagosomes and lysosomes by enhancing signal transduction in host cells.

\section{M. tuberculosis inhibits the acidification of phagolysosomes}

MTB inhibits the maturation of phagocytosis by suppressing the acidification of phagosomes and then persists in the relatively lower acidic environment ( $\mathrm{pH} 6.2$ ) (27). First, MTB inhibits phagosome acidification by changing its composition; the structure and specific molecules on the cell wall serve as a barrier, allowing the macrophages to maintain a neutral $\mathrm{pH}$ (27). Second, the protein phosphtase A (PtpA) downregulatesVATPase pump and plays direct role in the acid inhibition (28). Besides, infection of macrophages with MTB leads to the secretion of granulocyte-macrophage colonystimulating factor, triggering the expression of cytokine-inducible $\mathrm{SH} 2$-containing protein (CISH) through mediation by STAT5. VATPase catalytic subunit A can also 
ubiquitinate and degrade proteasomes by producing CISH.

\section{M. tuberculosis inhibits oxidative stress and the function of reactive oxygen and reactive nitrogen intermediates}

Oxidative stress is a disorder of pro/antioxidant balance, resulting in potential damage. Underlying the prolonged MTB latency in the host is not only the inhibition of macrophage phagocytosis, lysosome maturation and acidification but also the inhibition of oxidative stress.

ROS is highly toxic to bacteria as it can either directly destroy DNA, protein, and lipids or indirectly damage the nucleic acid via oxidation of the nucleotide pool (29). SigH,mshD, Wag31gene of mycobacterium protects it from heat, oxidative and nitric oxide stresses. The KatG and TrxB2 enzymes of MTB help to resist $\operatorname{ROS}(30)$. TRIM/TRAM pathway leads to the formation of TH2 cytokines which downregulates IL-12. IL-12 is required for the production of interferon- $\gamma$, iNOS, and NO, a major defense of the host against Mtb. The intracellular pathogen secretes SucB, AhpC, and AhpD, which catalyzes the breakdown of reactive nitric intermediates (RNIs).

\section{M. tuberculosis inhibits apoptosis and autophagy}

Invasion by MTB and host cell apoptosis require cellular factors, signaling proteins and regulation of the pathways involving TNF- $\alpha$, IFN- $\gamma$, transforming growth factors and IL-6, IL-12, IL-4 and IL-10 (31). NKT cells can produce and release IFN- $\gamma$ to inhibit the growth of MTB in macrophages (32). Macrophages infected with Mtb have upregulated IL-6 production, which selectively inhibits IFN- $\gamma$-induced autophagosome biogenesis. In addition, Mtb possess the "enhanced intracellular survival"
(Eis) gene, which attenuates autophagy and improves Mtb survival. Mtbup regulates the microRNAs miR-23a-5p, miR-20a, and miR33, which prevent the activation of autophagy. miRNAs are a class of noncoding small single-strand RNA molecules ( 22 nucleotides in length) that play a critical role in macrophage function. Overexpression of miR-30A suppresses the elimination of intracellular MTB and this is achieved by inhibiting autophagy.

In the conclusion we can say that upregulation of EIS gene and micro RNA and attenuation of IFN- $\gamma$ induced autophagosome biogenesis block autophagy and leads to Mycobacterium survival.

DCs and macrophages are the main components of the first line of defense against MTB and they can also maintain the complementary function of eliminating infectious bacteria (33). Surprisingly, infection by MTB is mainly based on cellular immunity, while the role of humoral immunity is controversial (34).

In conclusions, this review will systematically able us to understand why some genes are more expressed in active tuberculosis and why some of them are more expressed in the latent tuberculosis. Studying the above host pathogen interaction till date we can able to explore the potential of FYVE domain, Rab5GTPase, STAT-5, CISH, IL-10, MHC-II, MyD88, TLR, mir-30A, iNOS and many of the above discussed cytokines as a biomarker, to distinguish between active and latent tuberculosis in animals. In future we can also target more genes like PPAR $\gamma$ not studied yet for bovine tuberculosis. So in this review we studied the interaction of host macrophages and the pathogens, the immune response of host and its escape by the pathogen this will help us in the bovine tuberculosis diagnosis and treatment. 


\section{Acknowledgments}

Authors gratefully acknowledge financial support from the GADVASU Ludhiana.

\section{References}

1. Pavlik I, Ayele W, Havelkova M, Svejnochova M, Katalinic-Jankovic V, Zolnir-Dovc M. Mycobacterium bovis in human population in four central European countries during 1990 to 1999. Vet Med-Czech. 2003; 48(Suppl 4):90-98.

2. Smith NH, Gordon SV, De la RuaDomenech R, Clifton-Hadley RS, Hewinson RG. Bottlenecks and broomsticks: the molecular evolution of Mycobacterium bovis. Nat Rev Microbiol. 2006;4:670-681. doi: 10.1038/nrmicro1472.

3. Ameni GK, Tadesse E, Hailu Y, Deresse Y, Medhin G, Aseffa A, Hewinson G, Vordemeier M, Berg S. Transmission of Mycobacterium tuberculosis between farmers and cattle in Ethiopia. PLoS ONE. 2013;8(Suppl 10):e76891. doi: 10.1371/journal.pone.0076891.

4. Ocepek M, Pate M, Žolnir-Dovč M, Poljak M. Transmission of Mycobacterium tuberculosis from human to cattle. J. Clin. Microbiol. 2005; 43: 3555-3557. doi: 10.1128/JCM.43.7.35553557.2005.

5. Cosivi O, Grange JM, Daborn CJ, Raviglione MC, Fujikura $\mathrm{T}$, et al., (1998) Zoonotic tuberculosis due to Mycobacterium bovis in developing countries. Emerg Infect Dis 4: 59-70.

6. Ahmad S. Pathogenesis, immunology, and diagnosis of latent Mycobacterium tuberculosis infection. Clin Dev Immunol (2011) 2011:814943. doi:10.1155/2011/814943.

7. Gopalakrishnan A., Dietzold J., Verma S., Bhagavathula M., Salgame P. Toll-like receptor 2 prevents neutrophil-driven immunopathology during infection with Mycobacterium tuberculosis by curtailing CXCL5 production. Infect. Immun. 2018 doi: 10.1128/IAI.0076018.

8. Rohini K., Srikumar P.S. Insights from the docking and molecular dynamics simulation of the Phosphopantetheinyl transferase (PptT) structural model from Mycobacterium tuberculosis. Bioinformation. 2013; 9: 685-689. doi: 10.6026/97320630009685.

9. Cambier CJ, Takaki KK, Larson RP, Hernandez RE, Tobin DM, Urdahl KB, et al., Mycobacteria manipulate macrophage recruitment through coordinated use of membrane lipids. Nature (2014) 505:21822.doi:10.1038/nature12799

10. Kebir D, Jozsef L, Pan W, Wang L, Filep JG. Bacterial DNA activates endothelial cells and promotes neutrophil adherence through TLR9 signaling. J Immunol (2009) 182:4386-94. doi: 10.4049/jimmunol.0803044.

11. Almeida P. E, Carneiro A. B, Silva A. R, and Bozza P. T. PPAR $\gamma$ expression and function in mycobacterial infection: roles in lipid metabolism, immunity, and bacterial killing. PPAR Research, vol. 2012, Article ID 383829, 7 pages, 2012.

12. Harding CV, Boom WH. Regulation of antigen presentation by Mycobacterium tuberculosis: a role for toll-like receptors. Nat Rev Microbiol (2010) 8:296-307. doi:10.1038/nrmicro2321.

13. Saraav I, Singh S, Sharma S. Outcome of Mycobacterium tuberculosis and tolllike receptor interaction: immune response or immune evasion? Immunol Cell Biol (2014) 92:741-6. doi: 10.1038/icb.2014.52.

14. Mahajan S, Dkhar HK, Chandra V, Dave $\mathrm{S}$, Nanduri R, Janmeja $\mathrm{AK}$, et al., 
Mycobacterium tuberculosis modulates macrophage lipid-sensing nuclear receptors PPARgamma and TR4 for survival. J Immunol (2012) 188:5593603. doi:10.4049/jimmunol.1103038

15. Kleinnijenhuis J, Oosting M, Joosten LA, Netea MG, Van Crevel R. Innate immune recognition of Mycobacterium tuberculosis. Clin Dev Immunol (2011) 2011:405310. doi:10.1155/2011/405310.

16. Stamm CE, Collins AC, Shiloh MU. Sensing of Mycobacterium tuberculosis and consequences to both host and bacillus. Immunol Rev (2015) 264:20419. doi:10.1111/imr.12263

17. Noss EH, Pai RK, Sellati TJ, Radolf JD, Belisle J, Golenbock DT, et al., Tolllike receptor 2-dependent inhibition of macrophage class II MHC expression and antigen processing by $19-\mathrm{kDa}$ lipoprotein of Mycobacterium tuberculosis. J Immunol (2001) 167:910-8. doi:10.4049/jimmunol.167.2.910

18. Chang $\mathrm{CH}$, Guerder S, Hong SC, Van Ewijk W, Flavell RA. Mice lacking the MHC class II transactivator (CIITA) show tissue-specific impairment of MHC class II expression. Immunity (1996) 4:167-78.doi:10.1016/S10747613(00)80681-0

19. Ghorpade DS, Holla S, Sinha AY, Alagesan SK, Balaji KN. Nitric oxide and KLF4 protein epigenetically modify class II transactivator to repress major histocompatibility complex II expression during Mycobacterium bovis bacillus Calmette-Guerin infection. J BiolChem (2013) 288:20592606.doi:10.1074/jbc.M113.472183

20. Chang $\mathrm{CH}$, Flavell RA. Class II transactivator regulates the expression of multiple genes involved in antigen presentation. J Exp Med (1995) 181:765-7. doi:10.1084/jem.181.2.765
21. Pai RK, Pennini ME, Tobian AA, Canaday DH, Boom WH, Harding CV. Prolonged toll-like receptor signaling by Mycobacterium tuberculosis and its 19kilodalton lipoprotein inhibits gamma interferon-induced regulation of selected genes in macrophages. Infect Immun (2004) 72:6603-14. doi:10.1128/IAI.72.11.6603-6614.2004

22. Vergne I, Gilleron M, Nigou J. Manipulation of the endocytic pathway and phagocyte functions by Mycobacterium tuberculosis lipoarabinomannan. Front Cell Infect Microbiol (2014) 4:187. doi:10.3389/fcimb.2014.00187.

23. Malik ZA, Denning GM, Kusner DJ. Inhibition of $\mathrm{Ca}^{2+}$ ) signaling by Mycobacterium tuberculosis is associated with reduced phagosomelysosome fusion and increased survival within human macrophages. J Exp Med (2000) 191:287-302. doi:10.1084/jem.191.2.287.

24. Vergne I, Chua J, Deretic V. Tuberculosis toxin blocking phagosome maturation inhibits a novel $\mathrm{Ca} 2+/$ calmodulin-PI3K hVPS34 cascade. J Exp Med (2003) 198: 653-9. doi:10.1084/jem.20030527.

25.Guerin I, de Chastellier C. Pathogenic mycobacteria disrupt the macrophage actin filament network. Infect Immun (2000) 68:2655-62. doi:10.1128/IAI.68.5.2655-2662.2000

26. Podinovskaia M, Lee W, Caldwell S, Russell DG. Infection of macrophages with Mycobacterium tuberculosis induces global modifications to phagosomal function. Cell Microbiol (2013) 15:843-59. doi:10.1111/cmi.12092.

27. Chen Z., Wang T., Liu Z., Zhang G., Wang J., Feng S., Liang J. Inhibition of Autophagy by MiR-30A Induced by Mycobacteria tuberculosis as a Possible Mechanism of Immune Escape in 
Human Macrophages. Jpn. J. Infect. Dis. 2015; 68: 420-424. doi: 10.7883/yoken.JJID.2014.466.

28. Wong D., Li W., Chao J.D., Zhou P., Narula G., Tsui C., Ko M., Xie J., Martinez-Frailes C., Av-Gay Y. Protein tyrosine kinase, PtkA, is required for Mycobacterium tuberculosis growth in macrophages. Sci. Rep. 2018;8:155. doi: 10.1038/s41598-017-18547-9.

29. Acker Van H and Coenye T, "The role of reactive oxygen species in antibioticmediated killing of bacteria," Trends in Microbiology, vol. 25, no. 6, pp. 456466, 2017.

30. Lamichhane G. Mycobacterium tuberculosis response to stress from reactive oxygen and nitrogen species. Front. Microbiol. 2011; 2:176. doi: 10.3389/fmicb.2011.00176.

31. Liu M., Li W., Xiang X., Xie J. Mycobacterium tuberculosis effectors interfering host apoptosis signaling. Apoptosis. 2015; 20: 883-891. doi: 10.1007/s10495-015-1115-3.

32. Slight S.R., Khader S.A. Chemokinesshape the immune responses to tuberculosis. Cytokine Growth Factor Rev. 2013;24:105-113. doi: 10.1016/j.cytogfr.2012.10.002.

33. Dorhoi A., Kaufmann S.H. Versatile myeloid cell subsets contribute to tuberculosis-associated inflammation. Eur J. Immunol. 2015; 45: 2191-2202. doi: 10.1002/eji.201545493.

34. Schaller T., Ocwieja K.E., Rasaiyaah J., Price A.J., Brady T.L., Roth S.L., Hue S., Fletcher A.J., Lee K., KewalRamani V.N., et al., HIV-1 capsid-cyclophilin interactions determine nuclear import pathway, integration targeting and replication efficiency. PLoS Pathog. 2011; 7: e1002439. doi: 10.1371/journal.ppat.1002439.

\section{How to cite this article:}

Shalini Sharma and Gursimran Filia. 2020. Host Pathogen Interaction-Mycobacterium and Host Macrophages. Int.J.Curr.Microbiol.App.Sci. 9(02): 2415-2421. doi: https://doi.org/10.20546/ijcmas.2020.902.275 